\title{
Visiting Policies in the Adult Intensive Care Units in the Netherlands: Survey among ICU Directors
}

\author{
Kalinka Noordermeer, ${ }^{1}$ Tom A. Rijpstra, ${ }^{2}$ David Newhall, ${ }^{2}$ \\ Aline J. M. Pelle, ${ }^{3}$ and Nardo J. M. van der Meer ${ }^{2}$ \\ ${ }^{1}$ Department of Anaesthesiology, VU University Medical Centre, 1007 MB Amsterdam, The Netherlands \\ ${ }^{2}$ Department of Anaesthesiology and Intensive Care, Amphia Hospital, 4818 CK Breda, The Netherlands \\ ${ }^{3}$ Center of Research on Psychology in Somatic diseases (CoRPS), Tilburg University, 5037 AB Tilburg, The Netherlands
}

Correspondence should be addressed to Nardo J. M. van der Meer; nardo1@casema.nl

Received 2 June 2012; Accepted 25 June 2012

Academic Editors: M. Bailey, A. M. Japiassu, and S. L. Kane-Gill

Copyright (C) 2013 Kalinka Noordermeer et al. This is an open access article distributed under the Creative Commons Attribution License, which permits unrestricted use, distribution, and reproduction in any medium, provided the original work is properly cited.

Introduction. Admission to the ICU is a significant event for patients and their families and is often accompanied by stress, anxiety and depression. Literature shows that implementation of "unrestricted visiting policy" (UP) can potentially alleviate psychologically distressing elements of ICU admission. Methods. A web-based questionnaire was sent to all ICU's concerning three main topics: general ICU information, detailed visiting policy information, and rationale for the chosen policy. Results. $87.1 \%(n=74)$ of ICU's retain "restricted visiting policies" (RVP; $\leq$ five visiting hours per day). Knowledge about the current literature was overall $60.8 \%$. There is an UP in two academic hospitals and a "partly restricted policy" (PRP; $>5$ visiting hours per day but $<24$ ) in two academic, two large teaching and five general hospitals. Mean permissible duration in ICU's with a RVP was $165.6 \pm 79.2$ min versus $487.5 \pm 126$ min in the PRP. Conclusion. Nine out of ten ICU's still have a restricted visiting policy. The main reasons cited for a restricted visiting policy were potential interference with the daily clinical routine and privacy. A better knowledge of the current literature in combination with infrastructural changes might improve patients' outcome by reducing stress for the patient and its family.

\section{Introduction}

Patients and relatives often view Intensive Care Unit (ICU) admission as a significant step in medical treatment that is determined by the severity of a patient's condition. Under these circumstances the normal emotional response to hospital admission is exacerbated, therefore displacing both the patient and relatives to heightened levels of anxiety and stress. Although, the emotional significance and burden of an ICU admission is almost impossible to quantify, it has been proposed that an unrestricted visiting policy (UP) can benefit both the patients and families (UP; defined as a no restriction policy in time, duration of visitation and/or the number and kind of visitors). Theoretically, UP benefits patients and visitors by reducing stress and anxiety and therefore improves patient outcomes and visitors experience [1-7]. Although psychological quantitative data are difficult to correlate, Fumagalli et al. demonstrated a quantitative correlation between UP and improved outcome [3]. Their results highlighted a clear relationship between improved stress hormone levels and reduced cardiovascular complications. Furthermore, patients scored lower on depression scores, were less anxious, and felt more supported by relatives.

Nonetheless, skepticism surrounding UP still exists in Western Europe and it is therefore a reluctantly implemented policy [4-8]. Objections focus on disturbing the daily clinical routine, interfering with daily clinical practice, interrupting essential treatment and care, decreasing patient rest opportunities, and increasing infection rates in the patient population $[2,4-7]$. The net effect of these anecdotal theories is a worse outcome for patient recovery and a higher complication rate. However, there is no sound clinical literature that supports these theories. Furthermore, it has been demonstrated that despite an increased bacterial load in patients within UP ICUs, there was no increased infection or septic complication rate $[4,9]$. 
TABLE 1: Characteristics of the 85 responding ICU's* .

\begin{tabular}{lcc}
\hline & $n$ & Percentage \\
\hline Type of hospital & & \\
$\quad$ University teaching hospitals & 9 & $10.6 \%$ \\
$\quad$ Large teaching hospitals & 21 & $24.7 \%$ \\
$\quad$ General hospitals & 55 & $64.7 \%$ \\
Number of beds & & \\
$\quad \leq 6$ & 19 & $22.3 \%$ \\
$7-10$ & 22 & $25.9 \%$ \\
$>10$ & 44 & $51.8 \%$ \\
\hline
\end{tabular}

${ }^{*}$ Results are presented as $n(\%)$, unless otherwise indicated.

Using a web based questionnaire, our aim was not to investigate the conflicting evidence between the benefits and disadvantages of UP but instead to assess what policies are currently implemented in Dutch ICUs. Moreover, we wanted to understand the reasoning that the ICU had chosen their policy in terms of whether their decision was based on clinical or anecdotal evidence. Finally, we wanted to assess how the individual ICU had interpreted the available data and more fundamentally if they were aware of the current debate and data surrounding visiting policies.

\section{Materials and Methods}

2.1. Participants and Procedure. Following consultation with the Dutch institutional ethics committee, no ethical approval for our study was required. Over a two-week period (in May 2010), medical directors of all Dutch adult ICUs, including general and surgical ICUs, were contacted by telephone and informed of the study. In the event that a hospital had multiple ICUs, the ICUs were individually contacted. Overall directors of 102 ICUs were asked to participate. Oral permission and consent to participation were requested and followed two days later by a confirmation email that contained information about the study and a link to the online questionnaire. Each ICU had three weeks to submit their completed online questionnaire before a reminder email was sent. The database was closed ten weeks following the initiation of the study.

2.2. Instrument. A web-based online questionnaire was developed based on a literature review and a panel of experienced intensivists. Two further independent physicians reviewed the questionnaire for its content and clarity. The questionnaire consisted of 30 multiple-choice questions with two to ten answer options surrounding the following key themes: "general ICU information," "detailed visiting policy information" (including duration, number of visitors, and facilities), and "reasoning behind the chosen visiting policy including knowledge of current research.” The questionnaire required approximately 15 minutes to complete.

2.3. Statistical Analysis. We categorized visiting hour policies as "unrestricted" (UP) (visitors accepted at all times without restriction), "partly restricted policy" (PRP) (more than five hours per day but less than 24 hours per day) or a "restricted policy" (RVP) (five hours or less per day). In addition, details were collected on how many visitors were permitted per shift, visitor facilities, personnel provided to accompany visitors, and possibilities for adaptation in the current policy. Chisquare tests were used to examine differences in nominal variables and independent samples $t$-tests in continuous variables (Fisher exact tests and Mann-Whitney tests when appropriate). Statistical analysis was performed using PASW 17.0 (Chicago, Illinois, US), and a Pvalue $<0.05$ was used to denote statistical significance.

\section{Results}

The overall response rate of the ICU directors was $83.3 \%$. The characteristics of the participating ICUs are shown in Table 1. In $2.4 \%$ of the ICUs there was UP $(n=2)$, in $10.6 \%$ a PRP $(n=9)$ and in $87.1 \%$ an $\operatorname{RVP}(n=74)$. There was a significant difference $(P=0.001)$ in policy by type of hospital with UP only in two of the academic hospitals and not in the general or large teaching hospitals. Furthermore there is a PRP in $11.1 \%(n=2)$ of the academic hospitals, 9.5\% $(n=2)$ of the large teaching hospitals, and $7.2 \%(n=5)$ of the general hospitals. All the other ICUs had an RVP.

The mean permissible duration of visiting hours in the PRP was significantly longer than in the RVP $(P<$ 0.001). The mean permissible duration in the PRP was $487.5 \pm 126 \mathrm{~min}$ (range [300-600 minutes]), one missing value $(11.1 \%)$, whereas this was $165.6 \pm 79.2 \mathrm{~min}$ (range [60-420 minutes]), three missing values, (4.1\%) in the ICUs with RVP. Most RVP ICUs ( $n=46,62.2 \%)$ had visiting hours twice a day (Table 2 ). The estimated visiting time in the UP was $120 \pm 0$ min per day and $126.7 \pm 63.2 \mathrm{~min}$ per day (range [60-240]) in the PRP group. The majority of ICUs adapted their visiting hours in cases of "end-of-life" situations $(94.1 \%)$, or when patients were unstable $(85.9 \%)$. Other reasons for adaptation are named in Table 2. All ICUs had guidelines for the maximum number of visitors at one time (mean $2.1 \pm 0.5$ ). There were differences between the number of visitors allowed at any given time between the RVP, PRP, and UP, namely 2.1, 2.2, and 3.0, respectively $(P=0.05)$. Patients and first contacts reserved the right to restrict visitors in $72.3 \%(n=60)$ of ICUs. In $50.6 \%$ of all ICUs $(n=43)$ children were allowed, and of these ICUs $72.1 \%$ had no age limit. The facilities and support for family members of ICU patients are demonstrated in Table 3. Most ICUs offered facilities in a special family room $(82.4 \%)$ and in most ICUs there was a waiting room for visitors $(77.6 \%)$. Information about the patient's condition was shared with the family/first contact person by nurses (95.3\%), junior residents $(20.0 \%)$, and by appointment with the ICU consultant $(90.6 \%)$. In $12.9 \%(n=11)$ of ICUs there was a host(ess) to provide family support. Finally, most ICUs $(63.5 \%)$ do not take general precautions against infections while the patient was not in strict isolation. If any precautions were taken, the most common one reported was hand disinfection (34.1\%). A minority of the medical directors $(41.2 \%)$ reported to be familiar with the available 
TABLE 2: Characteristics of visiting policies and organization* ${ }^{*}$.

\begin{tabular}{|c|c|c|}
\hline & $n$ & Percentage \\
\hline \multicolumn{3}{|l|}{ Organization of visiting policy } \\
\hline Restricted & 74 & $87.1 \%$ \\
\hline Partly restricted & 9 & $10.6 \%$ \\
\hline Unrestricted & 2 & $2.4 \%$ \\
\hline \multicolumn{3}{|l|}{ Number of visiting periods a day } \\
\hline $1 x$ & 2 & $2.7 \%$ \\
\hline $2 \mathrm{x}$ & 45 & $60.8 \%$ \\
\hline $3 x$ & 26 & $35.1 \%$ \\
\hline$>3$ & 1 & $1.4 \%$ \\
\hline \multicolumn{3}{|c|}{$\begin{array}{l}\text { Possible visiting time in minutes per } \\
24 \mathrm{hr}(\text { mean } \pm \text { SD })^{\dagger}\end{array}$} \\
\hline $1 x$ & 240 & \\
\hline $2 \mathrm{x}$ & 137.1 & \pm 79.0 \\
\hline $3 x$ & 210 & \pm 56.1 \\
\hline Partly restricted & 487.5 & \pm 126 \\
\hline \multicolumn{3}{|l|}{$\begin{array}{l}\text { Adaptation of visiting hours if the } \\
\text { patient is: }\end{array}$} \\
\hline Unstable & 73 & $85.9 \%$ \\
\hline Dying & 80 & $94.1 \%$ \\
\hline Underage & 43 & $50.6 \%$ \\
\hline Awake and stable & 14 & $16.5 \%$ \\
\hline Delirious & 33 & $38.8 \%$ \\
\hline Fully sedated & 6 & $7.1 \%$ \\
\hline Other & 20 & $23.5 \%$ \\
\hline In isolation & 10 & $11.7 \%$ \\
\hline First day after admission & 3 & $3.5 \%$ \\
\hline
\end{tabular}

* Results are presented as $n$ (\%), unless otherwise indicated.

${ }^{\dagger}$ The total visiting time a day for the different number of visiting moments a day.

research that supports UP. Due to small numbers no statistical significant differences were found regarding awareness of current research (general hospitals $40 \%$, teaching hospitals $33.3 \%$, and academic hospitals $66.7 \%$ ). The most commonly cited argument against UP was the assumption that extended visiting hours would negatively interfere with daily clinical routine. RVP ICUs expected total visiting durations of 3 to 6 hours when changing their RVP to an UP. Additional arguments were a disturbance to the patient and a lack of privacy for the patient. The most frequently cited argument for UP was a "positive family experience".

Five ICUs reported a reversal of the UP to an RVP based on unacceptable disturbance levels for the patient and the ICU workers. In addition, one ICU cited unsuitable ward layout as the determining factor in adopting an RVP. Considerations for RVP, stratified by hospital type, are shown in Figure 1. The hospitals that use UP both reported an estimated mean daily visiting time duration of 2 hours $(120.0 \pm 0 \mathrm{~min})$.
TABLE 3: Facilities and support for visitors in the 85 responding ICUs (numbers and proportions).

\begin{tabular}{|c|c|c|}
\hline Characteristic & Value & \\
\hline \multicolumn{3}{|l|}{ Attention of visitors } \\
\hline Hostess & 10 & $11.9 \%$ \\
\hline Waiting room & 66 & $77.6 \%$ \\
\hline Family room & 70 & $82.4 \%$ \\
\hline Overnight stay & 15 & $17.6 \%$ \\
\hline Conversation room & 46 & $54.1 \%$ \\
\hline \multicolumn{3}{|l|}{ Infection prevention for visitors } \\
\hline No interventions & 54 & $63.5 \%$ \\
\hline Withdraw coat & 8 & $9.4 \%$ \\
\hline Disinfection hands & 29 & $34.1 \%$ \\
\hline Instruction by nurse & 1 & $1.2 \%$ \\
\hline \multicolumn{3}{|l|}{ Information supply (general) } \\
\hline Website & 37 & $43.5 \%$ \\
\hline Brochure & 81 & $95.3 \%$ \\
\hline Intake by hostess & 10 & $11.8 \%$ \\
\hline Intake by doctor & 49 & $57.6 \%$ \\
\hline Intake by nurse & 80 & $94.1 \%$ \\
\hline \multicolumn{3}{|c|}{ Information supply (patient related) } \\
\hline By the nurse & 81 & $95.3 \%$ \\
\hline By the resident on call & 17 & $20.0 \%$ \\
\hline By the ICU physician on call & 14 & $16.5 \%$ \\
\hline $\begin{array}{l}\text { Appointment with the ICU } \\
\text { physician }\end{array}$ & 77 & $90.6 \%$ \\
\hline Other & 12 & $14.1 \%$ \\
\hline
\end{tabular}

\section{Discussion}

Based on our research, we can report that collectively $41.2 \%$ of Dutch ICUs are aware of the literature surrounding visiting policies and that only $2.4 \%$ of the total number of Dutch ICUs have adopted UP based on a response rate of more than $80 \%$. Furthermore, five hospitals reverted back to an RVP following a trial period with an UP. These statistics are entirely consistent with research conducted across Europe including the United Kingdom (81.1\% RVP), Belgium (100\% RVP), France (97\% RVP), and Italy (99.6\% RVP) $[5,6,8,10]$ and reflect the reasons cited against UP in the literature. $[4,7,11]$ Recurrent objections to UP that transcended throughout the questionnaires included: interference with daily care, increased stress for the patient, and insufficient patient privacy. Interestingly, in contrast to European ICUs, infection risk was not cited as a decisive factor in not adopting UP. Furthermore, the highest proportion of PRP was found in academic hospitals (11.1\%) which arguably reflects that academic hospitals are more aware of the supporting literature for UP and PRP. The finding that $66.7 \%$ of academic hospitals were aware of the current supportive literature compared with $40 \%$ of general hospitals and $33.3 \%$ of teaching hospitals supports this theory.

We were moderately surprised by the sparsity of UP in the Netherlands owing to the generally supportive literature 


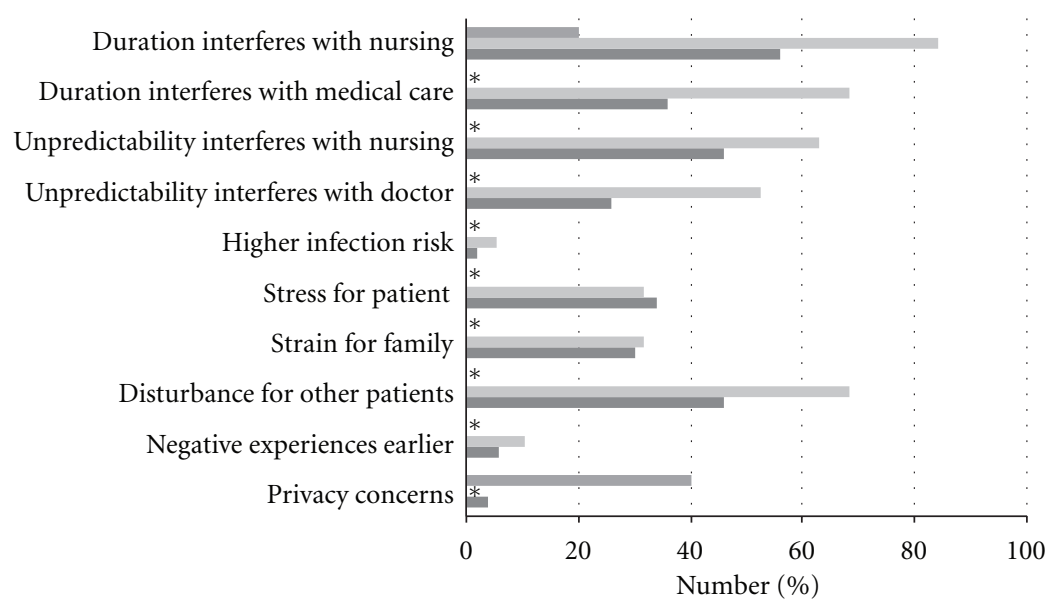

Figure 1: Different arguments used for an RVP by the Academic hospitals (dark grey color, $n=5$ ), Large teachings hospitals (grey color, $n=19$ ), and General hospitals (black color, $n=50$ ). ${ }^{*}$ No reason for RVP.

that surrounds this topic. An adoption of UP is generally regarded to increase the understanding and relationship between patients, family, and caretakers, and therefore in turn reduces levels of anxiety and depression [2, 3, 1216]. Evidence to underpin this theory is clearly provided by a small, randomized control trial (RCT) conducted by Fumagalli et al. [3]. Their RCT demonstrated that the risk of a cardiovascular event was two-fold higher in patients exposed to RVP as opposed to patients with UP. It was postulated that reduced stress and anxiety scores were a direct consequence of greater contact with friends and family. Moreover, favorable hormone profiling in the UP provided quantitative weight to their theory [3]. This research provides clear evidence supporting an adoption of UP.

Furthermore, great emphasis within the literature has also been placed on the advantages of a "family-centred" approach $[8,15,16]$. Nonetheless, only a minority of the ICUs offers overnight stay (17.6\%) and/or a specialized family host service (11.9\%). Effective and clear information at the point of admission is crucial in developing a fundamental understanding of the aims and goals of an ICU admission $[6,8,15,16]$. Familiarizing the relatives with the ICU setting theoretically reduces the stress and anxiety associated with a relative's admission. These feelings are then transposed to the patient, and the net gain is a patient with reduced stress and anxiety levels. Nevertheless, only $58.3 \%$ of all the responding Dutch ICUs have an admission meeting involving the medical staff. In $94 \%$ of ICUs there is an intake with a nurse and 95.2\% of ICUs supply written literature, such as brochures, as the primary source for delivering information regarding the ICU. Quantifying the benefit of clear communication with relatives at the point of admission is difficult to achieve and it was beyond the remit of this study. Nonetheless, in conjunction with our existing study, a patient and relative focused study could lend further weight to our theory that effective communication at the point of admission reduces relatives stress and anxiety. In turn, there is a synergistic beneficial effect on the patient.
Despite the evidence supporting UP, there remains considerable opposition to the adoption of UP as highlighted by the fact that only $2.4 \%$ of Dutch ICUs introduced such a policy. Main reasons mentioned in our survey were "an interference with the daily routine," "increased stress for the patient" and "patient privacy considerations." We found that there was little difference in the actual visiting times between UP and a PRP $120.0 \pm 0$ min per day and $126.7 \pm 63.2$ per day, respectively. Strikingly, five Dutch ICUs reversed their UP to a less open policy, for the reason of staff and/or patient convenience. This surprising fact, which has never been described, needs more attention in future research.

The key difference between UP and PRP was the freedom of movement allowed in the UP as the PRP usually comprised of two defined visiting blocks per day. The flexibility offered by UP is arguably of great benefit to the relatives, and therefore in turn the patient. However, what is not clear from our study is what time of day was most common for relatives in the UP ICU to visit and how this relates to a PRP or RVP. Furthermore, we have not investigated the effect of single or multiple rooms on the length of stay of relatives. This is relevant because a study among Dutch ICU nurses showed that single patient room ICUs had notably longer visiting hours [17]. This is a significant finding because the "Society of Critical Medicine" has clearly defined that private rooms are considered superior to multibed rooms as they offer more privacy and potentially lower infection risk [18]. Moreover, a single room facility would make the implementation of UP far easier and would nullify arguments against UP that are based on patient privacy. Nonetheless, they would however support the fear that in UP ICU with single bed facilities, very long visiting hours could be experienced [17-21].

In addition, to the aforementioned limitations, two others have to be mentioned also. Firstly, the study was limited to the medical directors of the ICUs across the Netherlands. Inclusion of senior nursing staff might have generated a more complete data set. Another limitation is the narrow range of choices in categorizing visiting hour policies (UP, PRP, 
and RVP). This timeframe categorization was formulated on the clinical experience of the study group. More timeframes might have given a clearer view on the total duration of visiting time.

\section{Conclusions}

Our results are consistent with results generated by previous research showing that almost nine out of ten ICUs employ a RVP. A fear of disrupting daily clinical routine with negative impact on patient care, privacy concerns, and an expected increase in visiting hours were the main reasons cited for a restricted visiting hours policy. Nonetheless, the reasons cited are not consistent with current literature. Furthermore, our data has shown that by adopting UP, the actual total daily visiting time does not increase significantly. Since the flexibility offered by UP have been shown to decrease stress and anxiety, facilitating UP, for instance, by increasing the number of single rooms combined with a "family-centered approach," might considerably benefit the patient's wellbeing.

\section{Abbreviations}

ICU: Intensive care unit

UP: Unrestricted visiting hours policy

PRP: Partly restricted visiting hours policy

RVP: Restricted visiting hours policy.

\section{Conflict of Interests}

The authors have no financial or nonfinancial conflict of interests.

\section{Contributions}

K. Noordermeer designed the study, developed the online questionnaire, made contact with the centers, did acquisition of data, and was involved in analysis and interpretation, drafting, and final approval. T. A. Rijpstra developed and/or approved the questionnaire, and was involved in analysis, interpretation of data, and final approval. D. Newhall drafted and critical revised the manuscript, final approval. A. M. Pelle designed the study, and was involved critical revising, statistical analysis, and final approval. N. J. M. van der meer designed the study, made contact with the centers, analysis and interpretation of the data, drafting and critical revising, and final approval.

\section{References}

[1] A. Giannini, "Open intensive care units: the case in favour," Minerva Anestesiologica, vol. 73, no. 5, pp. 299-306, 2007.

[2] C. E. Gonzalez, D. L. Carroll, J. S. Elliott, P. A. Fitzgerald, and H. J. Vallent, "Visiting preferences of patients in the intensive care unit and in a complex care medical unit," American Journal of Critical Care, vol. 13, no. 3, pp. 194-198, 2004.

[3] S. Fumagalli, L. Boncinelli, A. Lo Nostro et al., "Reduced cardiocirculatory complications with unrestrictive visiting policy in an intensive care unit: results from a pilot, randomized trial," Circulation, vol. 113, pp. 946-952, 2006.

[4] D. Berti, P. Ferdinande, and P. Moons, "Beliefs and attitudes of intensive care nurses toward visits and open visiting policy," Intensive Care Medicine, vol. 33, no. 6, pp. 1060-1065, 2007.

[5] A. Giannini, G. Miccinesi, and S. Leoncino, "Visiting policies in Italian intensive care units: a nationwide survey," Intensive Care Medicine, vol. 34, no. 7, pp. 1256-1262, 2008.

[6] J. D. Hunter, C. Goddard, M. Rothwell, S. Ketharaju, and H. Cooper, "A survey of intensive care unit visiting policies in the United Kingdom," Anaesthesia, vol. 65, no. 11, pp. 1101-1105, 2010.

[7] G. Biancofiore, L. M. Bindi, E. Barsotti, S. Menichini, and S. Baldini, "Open intensive care units: a regional survey about the beliefs and attitudes of healthcare professionals," Minerva Anestesiologica, vol. 76, no. 2, pp. 93-99, 2010.

[8] D. M. Vandijck, S. O. Labeau, C. E. Geerinckx et al., "An evaluation of family-centered care services and organization of visiting policies in Belgian intensive care units: a multicenter survey," Heart and Lung, vol. 39, no. 2, pp. 137-146, 2010.

[9] M. D. Lee, A. S. Friedenberg, D. H. Mukpo, K. Conray, A. Palmisciano, and M. M. Levy, "Visiting hours policies in New England intensive care units: strategies for improvement," Critical Care Medicine, vol. 35, no. 2, pp. 497-501, 2007.

[10] P. Quinio, C. Savry, A. Deghelt, M. Guilloux, J. Catineau, and A. de Tinténiac, "A multicenter survey of visiting policies in French intensive care units," Intensive Care Medicine, vol. 28, no. 10, pp. 1389-1394, 2002.

[11] L. Marco, I. Bermejillo, N. Garayalde, I. Sarrate, M. A. Margall, and M. C. Asiain, "Intensive care nurses' beliefs and attitudes towards the effect of open visiting on patients, family and nurses," Nursing in Critical Care, vol. 11, no. 1, pp. 33-41, 2006.

[12] M. Garrouste-Orgeas, F. Philippart, J. F. Timsit et al., "Perceptions of a 24-hour visiting policy in the intensive care unit," Critical Care Medicine, vol. 36, no. 1, pp. 30-35, 2008.

[13] J. S. Leske, "Treatment for family members in crisis after critical injury," AACN Clinical Issues, vol. 9, no. 1, pp. 129-139, 1998.

[14] J. A. Whitcomb, D. Roy, and V. S. Blackman, "Evidence-based practice in a military intensive care unit family visitation," Nursing Research, vol. 59, no. 1, pp. S32-S39, 2010.

[15] S. Verhaeghe, T. Defloor, F. Van Zuuren, M. Duijnstee, and M. Grypdonck, "The needs and experiences of family members of adult patients in an intensive care unit: a review of the literature," Journal of Clinical Nursing, vol. 14, no. 4, pp. 501-509, 2005.

[16] N. J. Henrich, P. Dodek, D. Heyland et al., "Qualitative analysis of an intensive care unit family satisfaction survey," Critical Care Medicine, vol. 39, no. 5, pp. 1000-1005, 2011.

[17] A. E. Spreen and M. J. Schuurmans, "Visiting policies in the adult intensive care units: a complete survey of Dutch ICUs," Intensive and Critical Care Nursing, vol. 27, no. 1, pp. 27-30, 2011.

[18] M. Rashid, "A decade of adult intensive care unit design: a study of the physical design features of the best-practice examples," Critical Care Nursing Quarterly, vol. 29, no. 4, pp. 282-311, 2006.

[19] M. Rashid, "Developing scales to evaluate staff perception of the effects of the physical environment on patient comfort, patient safety, patient privacy, family integration with patient care, and staff working conditions in adult intensive care units: a pilot study," Critical Care Nursing Quarterly, vol. 30, no. 3, pp. 271-283, 2007. 
[20] J. Bartley and A. J. Streifel, "Design of the environment of care for safety of patients and personnel: does form follow function or vice versa in the intensive care unit?" Critical Care Medicine, vol. 38, no. 8, pp. S388-S398, 2010.

[21] I. van de Glind, S. de Roode, and A. Goossensen, "Do patients in hospitals benefit from single rooms? A literature review," Health Policy, vol. 84, no. 2-3, pp. 153-161, 2007. 


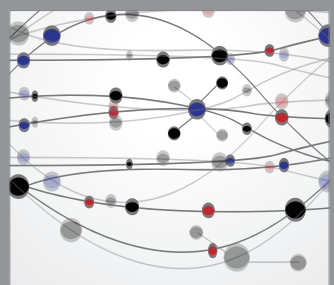

The Scientific World Journal
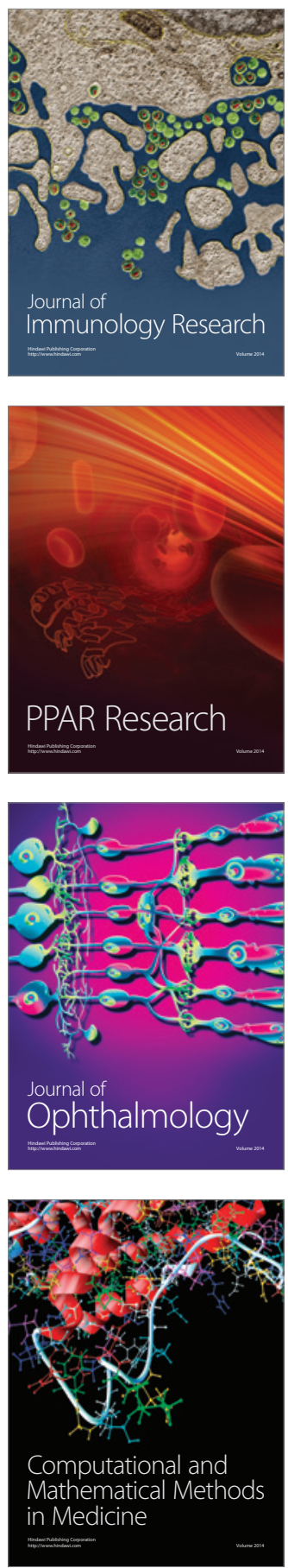

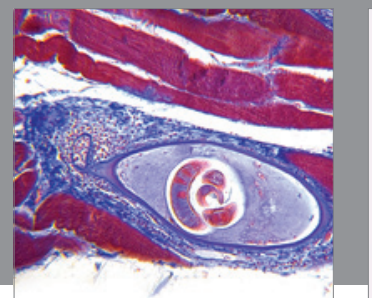

Gastroenterology

Research and Practice
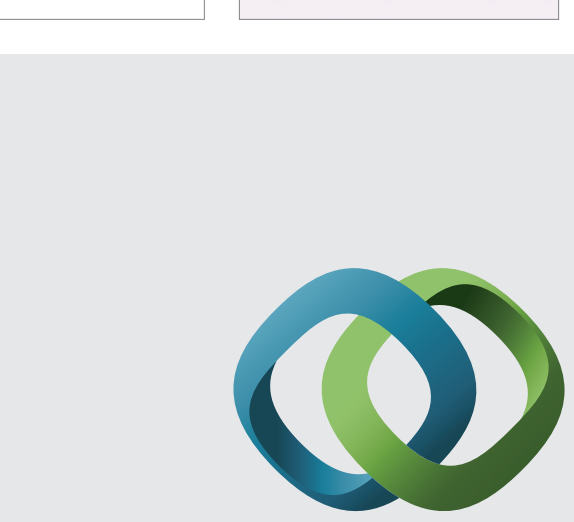

\section{Hindawi}

Submit your manuscripts at

http://www.hindawi.com
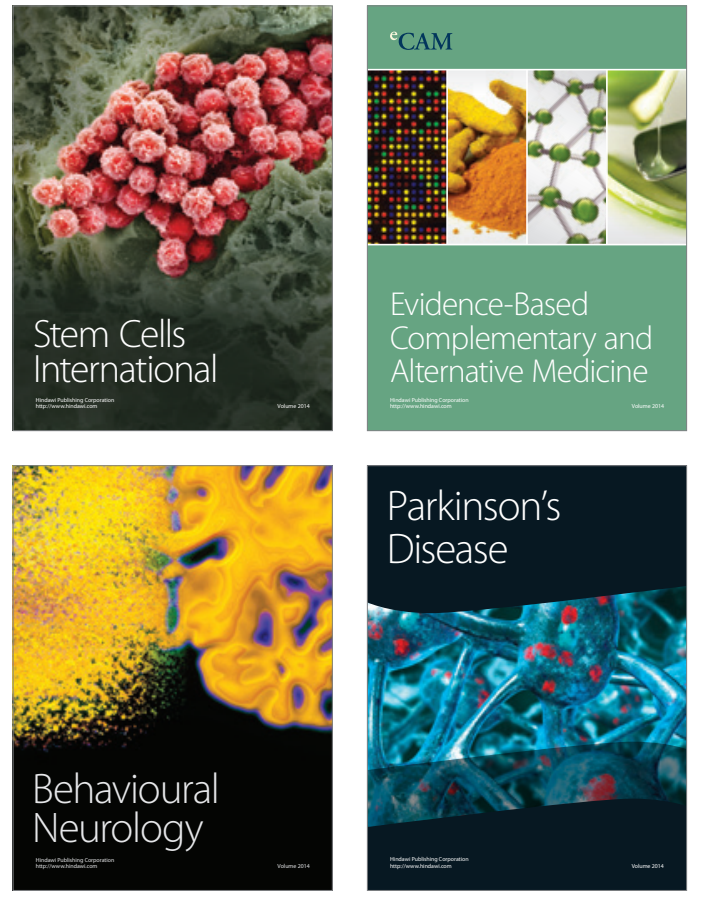
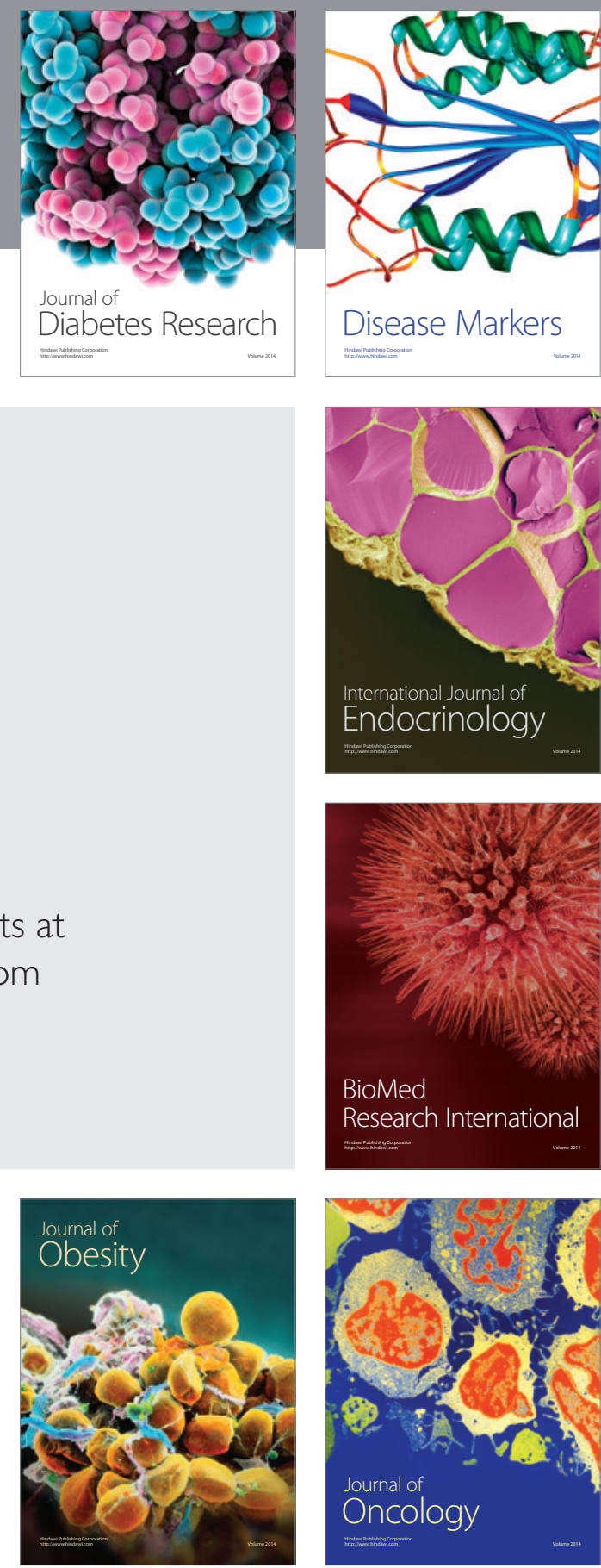

Disease Markers
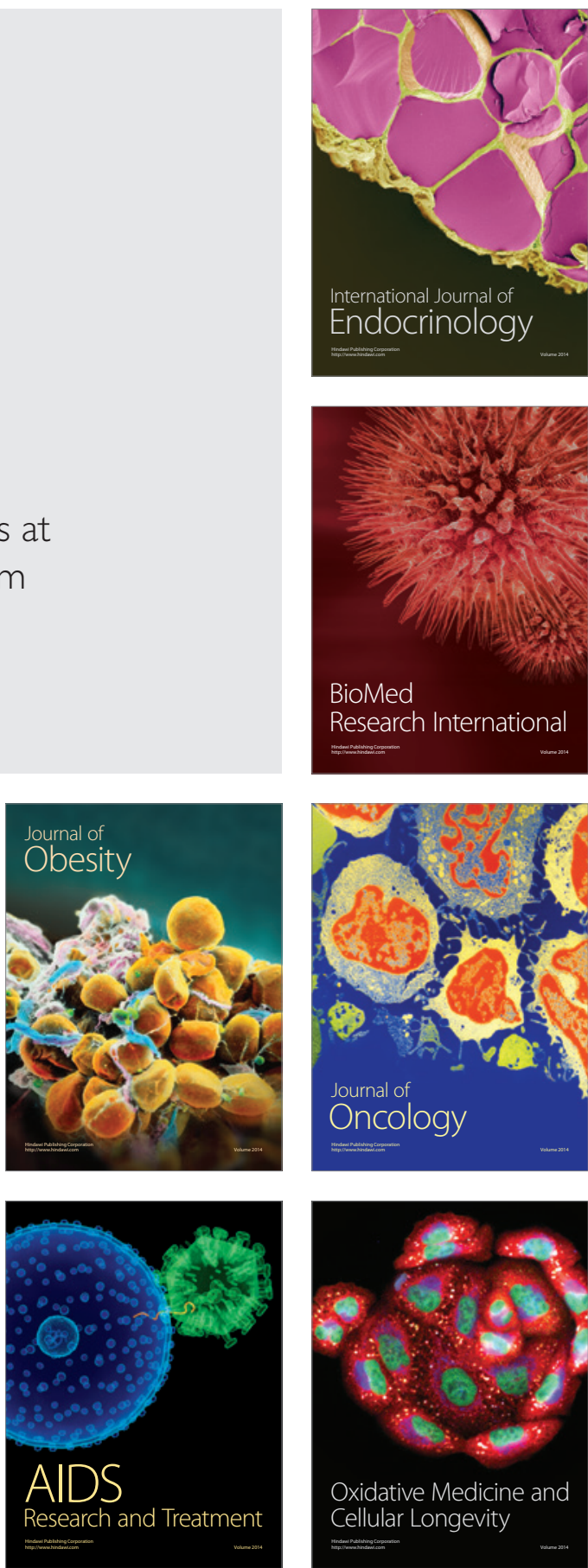